\title{
The prediction, diagnosis and management of complications in monochorionic twin pregnancies: the OMMIT (Optimal Management of Monochorionic Twins) study
}

Fiona L. Mackie ${ }^{1,2}$, R. Katie Morris ${ }^{1,2}$ and Mark D. Kilby ${ }^{1,2^{*}}$

\begin{abstract}
Background: Monochorionic twin pregnancies are at increased risk of complications due to sharing a single placenta and potentially developing unbalanced vascular anastomoses. Complications include twin-twin transfusion syndrome (TTS) which affects 10-15\% monochorionic twins, and if untreated has a 70-90\% perinatal loss rate. We are currently unable to predict which twins will develop complications or to what severity. We have previously shown differences in angiogenic and placental growth factors in maternal blood in pregnancies complicated by TTS compared to twin pregnancies not complicated by TTS but matched for gestation. There is also evidence to suggest that abnormal ultrasound measurements recorded in the first trimester (nuchal translucency and crown-rump length) may be associated with severe TITS later in pregnancy, however the detection rate is only reported as 52\%. We hypothesize that if these changes precede the development of the clinical syndrome, this may increase the sensitivity and specificity of detecting adverse pregnancy outcomes.
\end{abstract}

Methods: This cohort study has retrospective and prospective elements. In the retrospective cohort we will measure factors (decided based on preliminary work and a systematic review and meta-analysis) in stored maternal blood samples taken in the first-trimester, extract first-trimester ultrasound measurements and match these to pregnancy outcome. The prospective cohort will be divided into a "screening" cohort and "complicated" cohort. The screening cohort will undergo serial maternal blood sampling at 12, 16 and 20 weeks; we will extract ultrasound measurements and match to pregnancy outcome. The complicated cohort will comprise of women referred to the Fetal Medicine Centre with complications of monochorionicity. If the decision is taken to undergo fetoscopic laser ablation we will take maternal blood samples and amniotic fluid samples pre- and post-laser treatment. The same factors will be measured in the prospective cohort as informed by the retrospective study.

Discussion: This study aims to increase knowledge surrounding the pathology of complications in monochorionic twins, to aid future diagnosis and management.

Trial registration: ISRCTN 13114861 (retrospectively registered)

Keywords: Monochorionic, Twin-twin transfusion syndrome, Biomarker, Prediction, Fetoscopic laser ablation

\footnotetext{
* Correspondence: m.d.kilby@bham.ac.uk

${ }^{1}$ Centre of Women's and Newborn's Health \& Institute of Metabolism and

Systems Research, College of Medical and Dental Sciences, University of

Birmingham, Edgbaston, Birmingham B15 2TT, UK

${ }^{2}$ Fetal Medicine Centre, Birmingham Women's NHS Foundation Trust,

Edgbaston, Birmingham B15 2TG, UK
} 


\section{Background}

All twin pregnancies have increased risk over singletons, with monochorionic (MC) twins demonstrating the highest risk. Thirty per cent of twins are MC, but they experience $75 \%$ of all twin complications, the pathologies of which are closely linked to the fact that they share a single placenta and thus can develop unbalanced vascular anastomoses [1]. Complications that are specific to monochorionicity include twin-twin transfusion syndrome (TTTS), selective intrauterine growth restriction (sIUGR) and twin anaemia polycythaemia sequence (TAPS) [2]. TTTS complicates $10-15 \%$ of MC twins and usually occurs between 15 and 26 weeks. At present, the prognosis is dependent upon early treatment and method of treatment: if untreated it is associated with a perinatal loss rate of $70-90 \%$. A staging system, the Quintero system, exists but correlates poorly with outcome [3]. Even with apparently 'successful' antenatal treatment (with fetoscopic laser ablation (FLA)), TTTS is still associated with $10 \%$ neurological morbidity. We are currently unable to accurately predict which twins will develop complications or how severe they will be. There is some evidence that first trimester ultrasound screening of $\mathrm{MC}$ twin pregnancies between 11 and $13^{+6}$ weeks may predict adverse pregnancy outcome. These ultrasound features include: a) discordance in fetal nuchal translucency thickness (NT) with an increase in the recipient fetus $[4,5]$ and b) discordance in fetal crown rump length (CRL) measurements between the MC twins [5]. Although ultrasonographic features have a reasonable sensitivity and specificity in terms of allocating risk of severe TTTS, such screening is still associated with a significant false positive and false negative rate as the detection rate is only $52 \%$ [6].

We have recently reported significant differences in maternal serum between women with twin pregnancies complicated by TTTS, and twin pregnancies not complicated by TTTS but matched for gestational age [7]. These differences were seen in alpha-fetoprotein $(\alpha \mathrm{FP}), \beta$-hCG, plasma vascular endothelial growth factor (VEGF)-C, angiopoietin (Ang)-2 levels and the ratio of soluble vascular endothelial growth factor receptor-1 (sVEGFR-1) (also known as soluble fms-like tyrosine kinase-1. [sFlt-1]) to placental growth factor (PIGF) levels. We hypothesize that if these changes precede the development of the clinical syndrome, this may increase the sensitivity and specificity of detecting adverse pregnancy outcomes. The objective of this paper is to describe the aims and study design of our ongoing platform of work exploring complications in $\mathrm{MC}$ twin pregnancies.

\section{Methods}

\section{Aims of study}

The aim of our research is to improve knowledge of complications in MC twin pregnancies, and incorporate this knowledge into a predictive model in the future. Specifically we will investigate:

1. If abnormal first-trimester ultrasound measurements (NT and CRL) are associated with the development of complications later in pregnancy.

2. The effects that these complications and their treatment have on:

a. angiogenic factors in maternal serum/plasma

b. placental growth factors in maternal serum/plasma

c. angiogenic factors in amniotic fluid

d. placental growth factors in amniotic fluid

\section{Study design}

This cohort study has both retrospective and prospective elements. There will be 1 retrospectively recruited firsttrimester cohort, 1 prospectively recruited "screening" cohort, and 1 prospectively recruited "complicated" cohort. In all aspects of the work monochorionicity must have been confirmed using the ' $\mathrm{T}$ ' sign on first-trimester ultrasound scan.

\section{Retrospective cohort}

We will initially perform a retrospective study of stored maternal serum microbiology samples and Down Syndrome screening samples taken in the first trimester from MC twin pregnancies as part of routine booking at Birmingham Women's NHS Foundation Trust. These samples are routinely stored for 2 years in the microbiology and clinical chemistry department, and equate to approximately 170 samples. We will measure maternal angiogenic factors and placental growth factors in these samples, collect first trimester ultrasound measurements (CRL and NT) and patient characteristics and pregnancy outcome data. As Down Syndrome screening samples are received from 31 sites in the UK, we will ask clinicians at these sites to assist in data collection using a pre-designed proforma. Where clinicians feel unable to help with data collection, we will visit the sites and collect the outcomes ourselves. The primary outcome will be whether participants developed a complication in pregnancy (TTTS, TAPS, sIUGR, single or double intrauterine demise) and the severity of these complications. Secondary outcomes will include delivery (gestation at delivery, mode of delivery, birth weight, condition of twins at delivery) and neonatal outcomes (admission to neonatal unit, diagnosis of comorbidity, particularly neurological). The maternal angiogenic factors and placental growth factors measured will be decided based on our preliminary work (see above) and a systematic review and individual patient data (IPD) meta-analysis (see PROSPERO CRD42015024975 for full protocol). As this is a retrospective study and the results of our 
study will not affect patient care, patients whose blood samples we are using in this retrospective study will not be consented or informed of the results. However, all women under the care of Birmingham Women's NHS Foundation Trust are aware that the hospital is research active, and that their blood samples may be used in this way, and are given the option of opting out if they so wish. Samples from patients who have indicated that they do not want their samples to be used for research will be excluded from this study. This retrospective study will inform the subsequent prospective study.

\section{Prospective cohort}

The prospective study will run over 2 years (August 2015August 2017) and there will be 2 cohorts: a "screening" MC twin cohort (Cohort S), and a cohort who have developed complications (Cohort C) (see Fig. 1). In both cohorts, informed written consent will be obtained. The "screening" cohort (Cohort S) will be approached in the 'multiples' antenatal clinic where all twin pregnancies are managed at Birmingham Women's NHS Foundation Trust. We will ask participants with confirmed MC twin pregnancies to consent to 2 extra bottles of blood being taken during their routine Down Syndrome blood test or

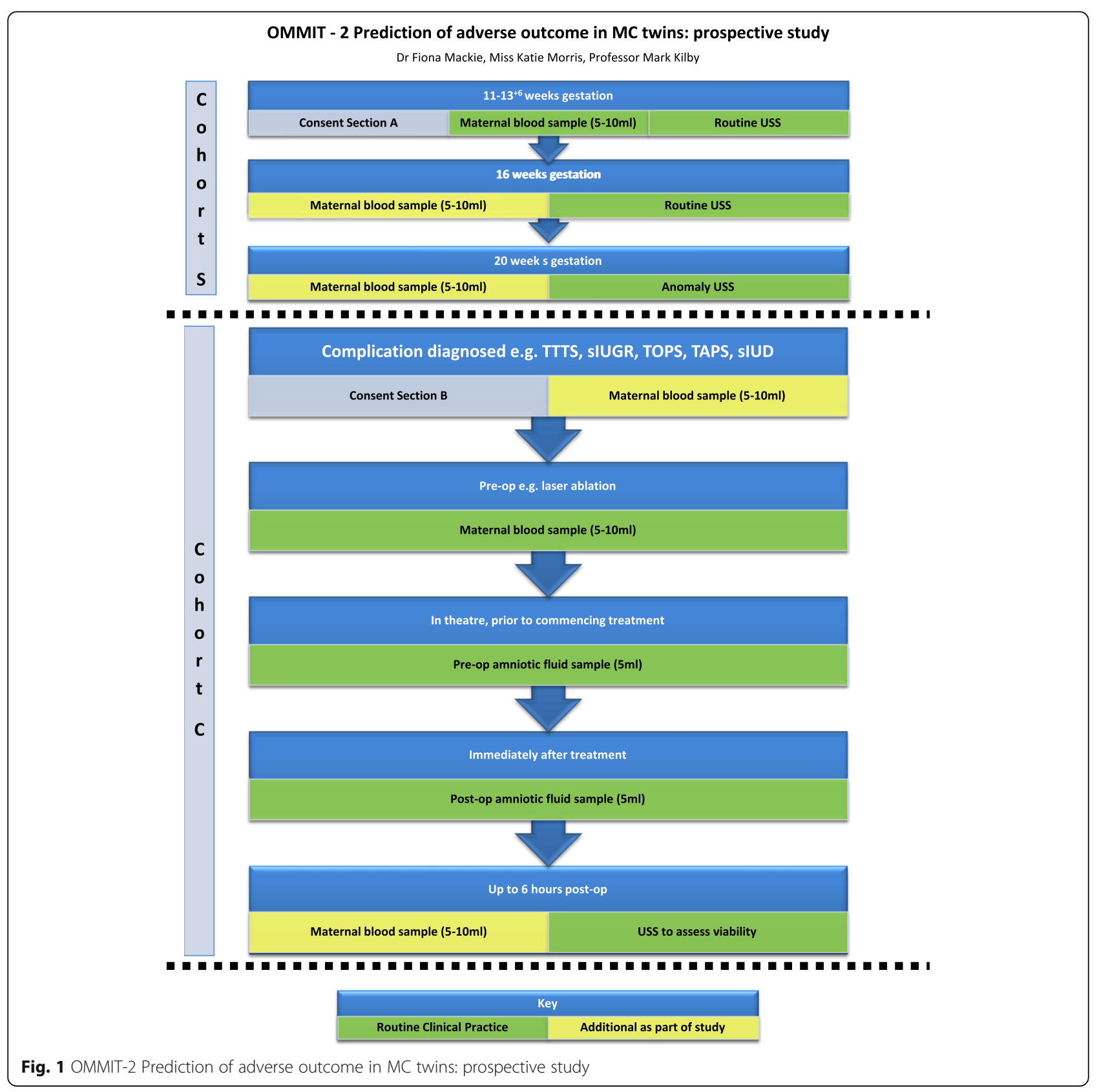


booking blood tests, and for permission to record their first trimester ultrasound measurements, and access the above outcome data as specified in the retrospective study. We will then repeat the blood sampling at 16 weeks and 20 weeks gestation when they attend for routine ultrasound scans. If the participant does not develop any complications, this will be the end of her involvement in the study and we will just collect the outcome data. However, if she develops a complication (e.g. TTTS, TAPS, sIUGR, single intrauterine demise) she will be invited and consented to enter the second prospective "complicated" cohort.

Women who develop complications are referred to the West Midlands Fetal Medicine Centre. The second cohort (Cohort C) will have serial maternal blood tests performed (pre-FLA and $6 \mathrm{~h}$ post-FLA) looking at the biomarkers informed by the IPD meta-analysis and retrospective study. A small amniotic fluid sample will be collected pre-FLA when the port for the fetoscope has been inserted into the recipient sac in the uterus, and a second small sample taken post-FLA when the ablation has been completed. The recipient twin will have polyhydramnios as it is part of the diagnostic criteria for TTTS, and it is routine clinical practice to perform amnioreduction at this point. All samples (serum and amniotic fluid) will be spun down, aliquoted, frozen and stored at $-80^{\circ}$ Celsius. We will assess the same factors in the amniotic fluid as in the maternal serum. Data will be collected on the ultrasound scans performed as part of routine clinical management of these conditions, as will pregnancy outcome data. Quintero staging will be used to classify TTTS [8].

\section{Expected number of participants}

In the retrospective study cohort, these samples are routinely stored for 2 years and equate to approximately 170 samples in total.

Regarding the prospective cohort S: Birmingham Women's NHS Foundation Trust has 7,900 deliveries per annum, approximately $2 \%$ of all maternities are multiple pregnancies and thus would equate to 158 maternities per annum. Of these, approximately 30\% are $\mathrm{MC}$ twins (47 MC twins per annum) and 70\% of women with an $\mathrm{MC}$ pregnancy will consent to Down syndrome screening (33 MC twins per annum). If we propose that 5 per year will be lost by miscarriage or decline participation in the study, then we predict recruitment of approximately $28 \mathrm{MC}$ twin pregnancies per annum, which equates to 56 maternities over 2 years of proposed recruitment.

At the West Midlands Fetal Medicine Centre we treat approximately 40-60 women a year with TTTS, which equates to 80-120 women over 2 years. If we allow for a $50 \%$ recruitment rate and incomplete follow-up, results in 40-60 sets of parents participating over 2 years in cohort $C$.

\section{Data management and data analyses}

At recruitment, each participant will receive a unique study identifier to allow linkage of data. Data will be collected using a purposely designed proforma (as per the retrospective cohort) and manually input onto a secure electronic database. Hard-copies of data will be kept in a locked filing cabinet, in a swipe-card access department at Birmingham Women's NHS Foundation Trust. We will ensure that we remain compliant with the Trust's Information Governance policies at all times.

Descriptive data will be reported. First trimester ultrasound measurements (NT and CRL) and serial serum biomarkers will be compared between participants in cohort $\mathrm{S}$ who go on to develop complications, and participants in cohort $S$ who do not develop complications. The serum biomarker results from the 16 and 20 week blood samples from participants in cohort $S$ will also act as the control group for cohort $\mathrm{C}$ participants. Additionally serum and amniotic fluid biomarkers will be compared longitudinally (i.e. pre- and post-FLA) for each participant in cohort $C$, to evaluate the potential effect of FLA on the biomarkers. The appropriate statistical tests will be used. Chi-squared will be used for categorical variables, and student's t-test (paired and unpaired where appropriate) for continuous variables. Logistic regression models will be created to assess the association between variables and outcomes and presented as odds ratios (OR) with a 95\% confidence interval (CI). Different cut-offs will be used for TTTS (Quintero staging) and sIUGR (EFW $<10^{\text {th }}$ centile, $<5^{\text {th }}$ centile).

\section{Discussion}

This study aims to increase knowledge surrounding the pathology of complications in MC twins, which in turn is hoped to aid future diagnosis and management. To our knowledge, this is the first study to look at these serum and amniotic fluid biomarkers in such a large cohort of $\mathrm{MC}$ twin pregnancies.

Although we are one of the largest Fetal Medicine Centres in the UK, the number of participants eligible for inclusion is insufficient to power the development of a prediction model, and may cause false negative results. The results of this study may inform a larger, multicentre study to be conducted over several years so as to create a prediction model to triage MC twin pregnancies as high-risk or low-risk for complications, and thus requiring more or less surveillance antenatally.

There will be some women who will have booked at Birmingham Women's NHS Foundation Trust and will be recruited to both the first-trimester screening cohort and the "complicated" cohort. Parents are routinely 
informed of the potential complications of MC pregnancy at booking, and by seeing these women in the first-trimester, this will give us the opportunity to inform them of the second part of the prospective study and they will have at least 4 weeks to consider participation should a complication develop. However, as a tertiary Fetal Medicine Centre we receive a lot of referrals from around the UK for second opinions and to perform treatment for $\mathrm{MC}$ twin complications, e.g. FLA. These referred patients will also be eligible for inclusion in the "complicated" cohort, although we will not have firsttrimester blood samples for them. Unfortunately, due to the rapid progression of complications, these parents may have a limited time to consider participation. However, this is still in-keeping with ethical approval.

Although we will be unable to gain consent for participants in the retrospective study, we intend to publish our results on the TAMBA website to which the public have access. We also hope to present them at international conferences and publish in leading medical journals.

We expect to lose some participants to follow-up, and to be unable to collect outcome data for some patients who may have moved areas, or have had their baby(ies) transferred out to different neonatal units.

\section{Conclusions}

This study hopes to increase knowledge of the pathological processes involved in the continuum of MC twin complications, and allow us to predict which MC twins will develop complications so that we are better able to target surveillance and therapy, and ultimately improve outcomes for these families.

\begin{abstract}
Abbreviations
Ang-2: Plasma angiopoietin; Cl: Confidence interval; Cohort C: "Complicated" cohort; Cohort S: "Screening" cohort; CRL: Crown rump length; EFW: Estimated fetal weight; FLA: Fetoscopic laser ablation; IPD: Individual patient data; MC: Monochorionic; NT: Nuchal translucency; OR: Odds ratio; PAPPA: Pregnancy associated plasma protein A; PIGF: Placental growth factor; sFLT-1: Soluble fms-like tyrosine kinase-1; sIUGR: Selective intrauterine growth restriction; SVEGFR-1: Soluble vascular endothelial growth factor receptor-1; TAPS: Twin anaemia polycythaemia sequence; TTTS: Twin-twin transfusion syndrome; VEGF-C: Plasma vascular endothelial growth factor; aFP: Alpha feto-protein; $\beta$-hCG: Beta human chorionic gonadotrophin
\end{abstract}

\section{Acknowledgements}

This work is funding by the Richard and Jack Wiseman Trust who have peer-reviewed this work.

\section{Funding}

This work is funded by the Richard and Jack Wiseman Trust. The funding body peer-reviewed the study protocol but is not involved in the study is any other way.

\section{Availability of data and materials}

Datasets will be presented in the main paper, as additional supporting files, or in publicly available repositories (where available and appropriate).

\section{Authors' contributions}

FLM, RKM and MDK are in the core research team conducting this research. MDK and RKM conceived the idea for the manuscript, FLM and RKM drafted the manuscript, MDK made final editorial decisions regarding the manuscript. All authors read and approved the final manuscript.

\section{Competing interests}

The authors declare that they have no competing interests.

\section{Consent for publication}

Not applicable.

\section{Ethics approval and consent to participate}

This research has been granted ethical approval by East Midlands REC Committee (15/EM/0240) and the Health Research Authority (15/CAG/0142) in June 2015 for the retrospective work. The prospective work has been granted ethical approval by East Midlands REC Committee (15/EM/0244) in July 2015, and the $1^{\text {st }}$ amendment was approved in August 2015.

\section{Publisher's Note}

Springer Nature remains neutral with regard to jurisdictional claims in published maps and institutional affiliations.

Received: 26 February 2016 Accepted: 19 May 2017

Published online: 26 May 2017

References

1. Sebire NJ, D'Ercole C, Hughes K, Carvalho M, Nicolaides KH. Increased nuchal translucency thickness at 10-14 weeks of gestation as a predictor of severe twin-to-twin transfusion syndrome. Ultrasound Obstet Gynecol. 1997;10(2):86-9.

2. Crowther $C$. Multiple pregnancy. In: James D, Steer $P$, Weiner $C$, Gonik B, editors. High risk pregnancy. 2nd ed. London: WB Saunders; 2001. p. 129-51.

3. Rossi A, D'Addario $V$. The efficacy of Quintero staging system to assess severity of twin-twin transfusion syndrome treated with laser therapy: a systematic review with meta-analysis. Am J Perinatol. 2009;26:537-44

4. Sebire NJ, Souka A, Skentou H, Geerts L, Nicolaides KH. Early prediction of severe twin-to-twin transfusion syndrome. Hum Reprod. 2000;15(9):2008-10.

5. El Kateb A, Nasr B, Nassar M, Bernard JP, Ville Y. First-trimester ultrasound examination and the outcome of monochorionic twin pregnancies. Prenat Diagn. 2007;27(10):922-5.

6. Kagan KO, Gazzoni A, Sepulveda-Gonzalez G, Sotiriadis A, Nicolaides KH. Discordance in nuchal translucency thickness in the prediction of severe twin-to-twin transfusion syndrome. Ultrasound Obstet Gynecol. 2007; 29(5):527-32.

7. Fox CE, Pretlove SJ, Chan BC, Mahony RT, Holder R, Kilby MD. Maternal serum markers of placental damage in uncomplicated dichorionic and monochorionic pregnancies in comparison with monochorionic pregnancies complicated by severe twin-to-twin transfusion syndrome and the response to fetoscopic laser ablation. Eur J Obstet Gynecol Reprod Biol. 2009:144(2):124-9.

8. Quintero R, Morales W, Allen M, Bornick O, Johnson P, Kruger M. Staging of twin-twin transfusion syndrome. J Perinatol. 1999;19:550-5.

Submit your next manuscript to BioMed Central and we will help you at every step:

- We accept pre-submission inquiries

- Our selector tool helps you to find the most relevant journal

- We provide round the clock customer support

- Convenient online submission

- Thorough peer review

- Inclusion in PubMed and all major indexing services

- Maximum visibility for your research

Submit your manuscript at www.biomedcentral.com/submit 